\title{
Ovarian cancer: diagnostic accuracy and tumor types distribution in East Africa compared to North America
}

\author{
Peter F. Rambau ${ }^{1 *} \mathbb{D}$, Martin Köbel ${ }^{2}$, Derek Tilley ${ }^{3}$, Alex Mremi ${ }^{4}$, Robert Lukande ${ }^{5}$ and William Muller ${ }^{6}$
}

\begin{abstract}
Background: Ovarian cancer is a spectrum of several histologically distinct tumor types that differ in etiology, response to therapy, and prognosis. In resource-limited settings, the diagnosis of ovarian cancer can be challenging. This study describes the distribution of ovarian cancer tumor types in East Africa as well as assessing the diagnostic accuracy by using contemporary methods.

Methods: Data from 210 women identified from the records with a diagnosis of ovarian cancer in a period of 15 years were included. Two tissue microarrays were constructed and stained with 20 antibodies relevant to ovarian cancer subtyping. An integrated diagnosis was reached by the review of full Haematoxylin and Eosin stained sections, with consideration of immunohistochemical results. The integrated diagnoses were compared with the original diagnoses, and the degree of agreement was evaluated by percentage and Kappa statistics.

Results: Though limited by selection bias, the results suggest lower rates of ovarian cancer in East Africa compared to a North American population from Alberta, Canada. There was a higher proportion of sex cord stromal tumors and germ cell tumors in the East African population. Diagnostic accuracy for main ovarian tumor type categories was substantial (Kappa 0.70), but only fair for specific ovarian carcinoma histotypes (Kappa 0.34). Poor Haematoxylin and Eosin stain was the main factor hindering the correct diagnosis, which was not related to tissue processing.

Conclusions: In a resource-limited setting, where immunohistochemistry is not routinely carried out, diagnostic accuracy for the main categories of ovarian carcinoma is substantial and could be further improved by standardization of the basic Haematoxylin and Eosin stain.
\end{abstract}

Keywords: Ovarian cancer, Histotypes, Immunohistochemistry, Diagnostic accuracy

\section{Background}

There is considerable regional variation in the incidences of ovarian cancer, whereby the highest rates are seen in Europe (10.1 per 100,000) and North America (8.7 per $100,000)$. In contrast, the lowest incidences are seen in Japan and developing countries [1]. Data for Africa are limited as there are fewer cancer registries and cancer

\footnotetext{
* Correspondence: ra1972tz@yahoo.com

1 Department of Pathology, Catholic University of Health and Allied Sciences (CUHAS), Box 1464, Mwanza, Tanzania

Full list of author information is available at the end of the article
}

control programmes. In Africa, cancer statistics are based mainly on estimates [2,3], which show a lower rate of 4.2 per 100,000. In Sub-Saharan Africa, the burden and types of ovarian cancer are largely unknown. In the East African countries, data from Kenya shows that ovarian cancer constitutes $3.4 \%$ of all cancers. However, these data are based on cancer registries located in major urban cities and do not include the rural population [4].

The global distribution of ovarian cancer tumor types has been documented, and the majority of ovarian 
malignancies are epithelial [5]. Ovarian cancers are further subtyped into histotypes, which proved to be predictors for treatment choice, prognostication, and genetic counselling. Correct diagnosis and typing are, therefore, becoming mandatory [6]. Historically, there has been misclassification of ovarian carcinoma [6], with the African data considered unreliable, and the influence of local diagnostic practice is unknown. The cancer burden in African countries is increasing [7], and health facilities are facing significant challenges in many SubSaharan African countries [3]. Pathology services in Africa show significant gaps of qualified professional and technical staff, inadequate infrastructure, and low operational funding [8-10]. Cancer treatment rests on an accurate diagnosis, which represents a major challenge for the increasing number of cancer patients.

This study aimed to assess the frequency and diagnostic accuracy of understudied ovarian cancer types in selected centers in Tanzania and Uganda, compared to a population from Alberta, Canada.

\section{Methods}

\section{Study sites}

This retrospective study involved cases of ovarian cancer diagnosed in the year 2002 to 2017 from three centers in Tanzania: Bugando Medical Centre (BMC), Kilimanjaro Christian Medical Center (KCMC), and Mbeya Referral Hospital, and the Pathology Department at Makerere University, Kampala, Uganda. These are the only tertiary hospitals with diagnostic facility for cancer, all patients or samples of patients with cancer are brought to these centers for diagnosis. Bugando Medical Centre serves the North-Western area of Tanzania with a population of 13 million; KCMC serves a population of 11 million from the North-Eastern part of Tanzania; and the Mbeya Referral Hospital serves a population of 8 million from the Southern Highlands of Tanzania. In Uganda, the Pathology Department of Mulago Hospital serves a population of 3 million.

\section{Pathological and clinical information}

A search was undertaken for cases of ovarian cancer diagnosed from 2002 to 2017 from the files of the pathology department at each participating center. For each ovarian cancer diagnosis identified, the histological type was recorded as well as the demographic characteristics of the patient. This process was completed by searching the medical records from the patient files. By using the histology number, a corresponding formalin fixed paraffin embedded (FFPE) tissue block and haematoxylin and eosin (H\&E) stained histological slides were retrieved from the archival pathology materials. In circumstances where the $H \& E$ stained slides were not available, new sections were made and stained by $\mathrm{H} \& \mathrm{E}$. All $\mathrm{H} \& \mathrm{E}$ stained slides were examined under a light microscope, and the slides with a viable representative tumor were selected and matched with the corresponding FFPE tissue block. In each case, one to three H\&E stained slides and FFPE tissue blocks were selected for the study. The selected FFPE tissue blocks and corresponding H\&E stained slides were shipped to the Anatomic Pathology Research Laboratory (APRL) of the University of Calgary, Alberta, for further studies.

\section{Tissue microarray (TMA) construction}

In each case, one good representative H\&E stained slide was selected and marked for an area with high tumor cellularity. The corresponding area on the FFPE tissue block was selected and punched with a $0.6 \mathrm{~mm}$ needle core for TMA construction. Two tissue microarrays containing $0.6 \mathrm{~mm}$ duplicate cores from each case were constructed. This procedure was carried out at the Anatomical Pathology Research Laboratory (APRL) of the University of Calgary by a Semi-automated Tissue Arrayer (TMArrayer ${ }^{\mathrm{Tw}}$, Pathology Devices, Inc. USA).

\section{Immunohistochemistry (IHC)}

Antigen retrieval, staining platforms, antibody dilutions, detection methods, and interpretation of the staining are shown in Supplementary Table S-1. In brief, 0.4- $\mu \mathrm{m}$ sections were made from the two TMA's, and staining was carried out by the Dako Omnis Platform of the Calgary Laboratory Services, and the APRL of the University of Calgary. The TMA's were stained with the following antibodies: antibodies for general interest in ovarian cancer AE1/AE3 and PAX8, and antibodies specific for ovarian carcinoma typing as described previously [11]: WT-1, p53, Napsin A, PR, p16, TFF3, ARID1A, and Vimentin. More antibodies to complement typing for ovarian carcinoma were added: ER, Mismatch repair proteins (MSH6 and PMS2) and SATB2. For germ cell tumors and Sex-cord Stromal tumors, the following antibodies were included: Inhibin, FoxL2, Melan A, OCT-4, and GLYP3. CD45 and c-myc were used for lymphomas as well as BRG1 for small cell carcinoma hypercalcemic type.

\section{Histotype assignment}

Full section H\&E stained slides from each ovarian cancer cases were reviewed under light microscopy by the investigators. The morphology of ovarian cancer was assessed based on the 2014 WHO classification of tumors of the female reproductive tract and complemented by immunohistochemical expression patterns as previously described [11]. In a situation where there was discordance between morphology and immunophenotype, a consensus was reached by the arbitration of a third reviewer. 


\section{Alberta cancer registry}

We identified all patients diagnosed with ovarian cancer in Alberta between 2008 and 2016 from the Alberta Cancer Registry. Patient demographics and treatment data were extracted from the Discharge Abstract Database, the National Ambulatory Care Reporting System Database, or were obtained directly from electronic medical records (ARIA RO/MO).

\section{Statistical analysis}

Data was entered and cleaned using a Microsoft Excel spreadsheet, and the analysis was performed with statistical software STATA 13 (College Station, TX, USA) and JMP12 (SAS, Cary, NC, USA). For analysis, categorical variables were summarized as the proportions, and continuous variables were summarized as means with standard error. The statistical tests were performed by two-sided Chi-square test for categorical data and twosided student-t tests for the continuous data. The statistically significant differences were considered when $p$ values were less than 0.05 . Agreement between original diagnosis and the revised diagnosis was assessed as a percentage of cases agreed upon (concordance), and by Cohen's kappa.

\section{Results}

Study composition and patients' characteristics

A total number of 371 patients with a diagnosis of ovarian cancer made between 2002 and 2017 were identified from the study sites. Following exclusions, two TMA's were constructed representing 227 ovarian cancer cases. Further exclusions resulted in 210 ovarian cancer cases suitable for analysis, as shown in Fig. 1. For all patients enrolled, the median age was 47.5 (Range 6 to 86) years. The patients presented with various symptoms and the most common symptoms were abdominal swelling, abdominal pain, abdominal distention, and abnormal uterine bleeding. The duration of the condition ranged from 2 weeks to 84 months, with a median duration of 6.5 months.

Menopausal status was available for 91 patients only, whereas 34 (37.4\%) were post-menopausal. All patients underwent surgery, and the majority of the specimens received for investigation were ovarian masses 126 (60\%), followed by hysterectomy and bilateral salpingooophorectomy. In a few cases, staging surgery was accompanied by omentectomy, bowel resection, and lymph node sampling. The stage of the disease was available in four patients only, and all were at an advanced stage. There was no data on disease recurrence or survival.

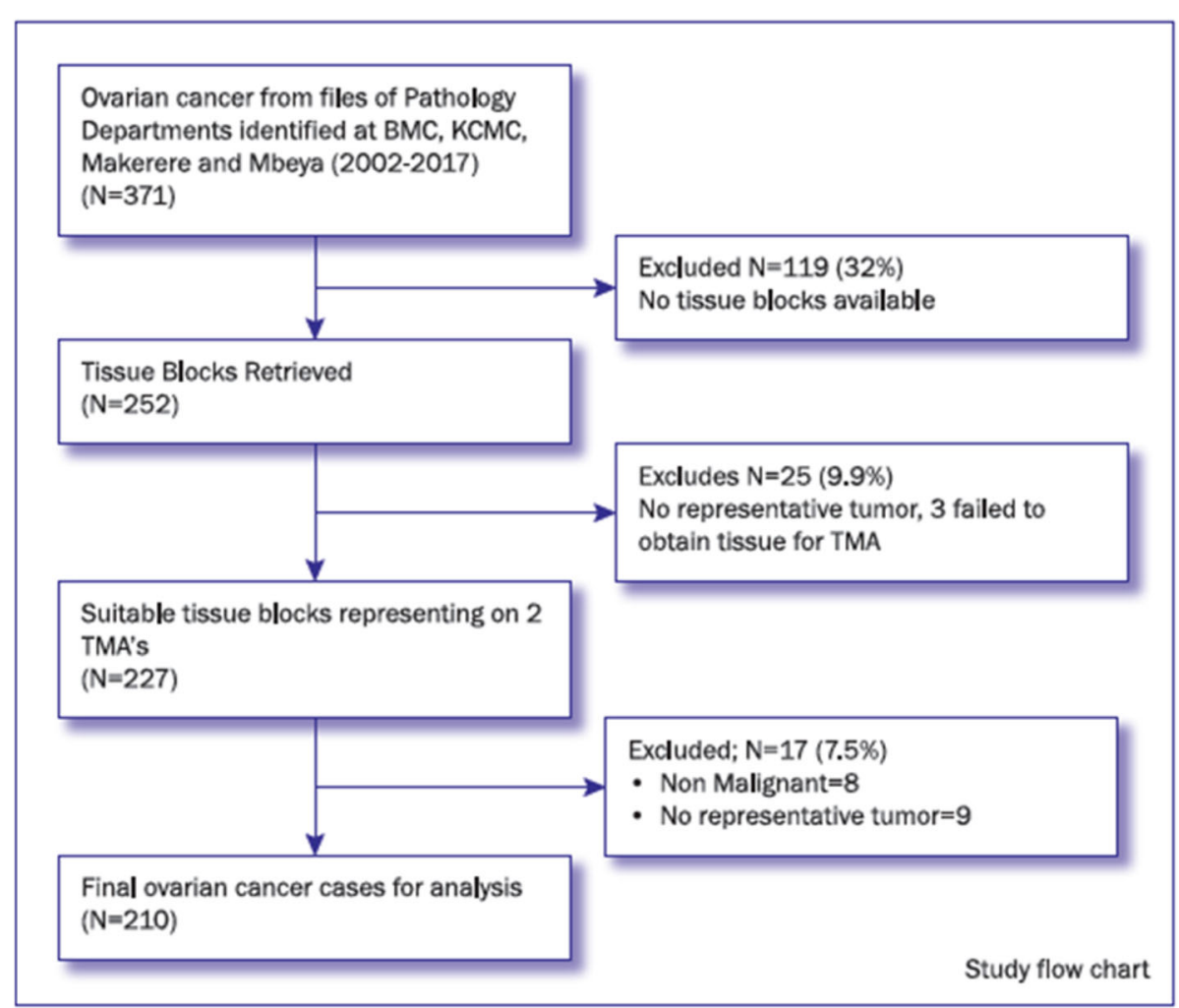

Fig. 1 Study flow chart 
Ovarian tumor types in original and revised diagnoses

In the original diagnosis, the majority of ovarian cancers were epithelia (carcinomas), which constituted 147 (70\%) of all the cases with a mean age of 50.5 ( $\mathrm{SE} \pm 1.2$ ) years. The second most common diagnosis was a sex cord stromal tumor in $24(11.4 \%)$ cases with a mean age of 37 ( $\mathrm{SE} \pm 3.0$ ) years, followed by germ cell tumors 21 $(10 \%)$, with a mean age of $22.8(\mathrm{SE} \pm 3.2)$ years. The diagnosis of ovarian lymphomas was rendered in 14 $(6.7 \%)$ of the cases with a mean age of $15.9(\mathrm{SE} \pm 3.7)$ years. Other types included sarcoma in $2(0.9 \%)$ cases and unspecified cancers in $2(0.9 \%)$ cases.

Following morphological review and IHC integration, there was substantial agreement between original diagnosis and a revised diagnosis in the major categories of ovarian cancer (Kappa 0.702 with 95\% CI of 0.619 to 0.791 ), as shown in Table 1. In a revised diagnosis, the largest category was still epithelia in 127 (60.5\%) of all the cases with a mean age of $52(\mathrm{SE} \pm 1.2)$ years. The second most common category was sex cord stromal tumors with 30 (14.3\%) cases with a mean age of 45 (SE \pm $2.9)$ years, followed by germ cell tumors 27 (12.6\%), and lymphomas 13 (6.2\%). There was no significant mean age difference from the original to the revised diagnosis between ovarian tumor types.

\section{Histotype specific agreement between original diagnosis and revised diagnosis}

In a revised diagnosis, the specific histotypes were assigned based on 2014 WHO Classification of Tumors of Female Reproductive Organs. There was a fair agreement between original diagnoses and revised diagnoses (Kappa $=0.343$, 95\% CI: 0.277 to 0.409 ), as shown in Supplementary Table 2. In summary, a good concordance was seen in Lymphomas, followed by Germ Cell Tumors (92.9 and 81\%, respectively). For epithelial tumors (carcinoma), a total number of 84 (57.9\%) cases were not classified (carcinoma not otherwise specified (NOS)). Following review, a total number of $36(42.9 \%)$ cases were reclassified to High Grade Serous Carcinoma (HGSC). Significant numbers of carcinoma NOS had poor H\&E stains, in which the diagnosis was straightforward following recut and new H\&E stain at Calgary laboratory. Two cases had a diagnosis of carcinoma NOS. Following H\&E re-stain, one was adult granulosa cell tumor, and the other was HGSC with positive staining with WT-1 and p53 mutant type (see Fig. 2). There were 13 cases of HGSC in the original diagnosis, and the concordance with the revised diagnosis was $76.9 \%$. Among 17 cases originally diagnosed as Endometrioid Carcinoma (EC), the concordance with a revised diagnosis was $58.8 \%$, and 5 (29.4\%) were reclassified to HGSC. The concordance of $21.7 \%$ was seen in $\mathrm{Mu}-$ cinous Carcinoma (MC), and 6 (26.1\%) were reclassified to EC, whereas 4 (17.4\%) were metastasis from GI. There were three cases of Low Grade Serous Carcinoma (LGSC), with a concordance of $33.3 \%$, one case was EC, and the other was a metastasis from GI. There was one case of neuroblastoma which was reclassified as small cell carcinoma hypercalcemic type (SCCOHT) with loss of BRG1 staining in tumor cells and positive staining of stromal cells (See Fig. 3a \& b), and one case of carcinoma NOS was reclassified as mixed carcinoma (EC/LGSC).

\section{IHC markers expression in ovarian cancers}

The patterns of IHC markers expression across different ovarian cancer histotypes are shown in Table 2 . PAX8 was expressed in all LGSC, in $87 \%$ of HGSC and in none of the mucinous carcinomas. The Fallopian tube marker WT-1 was positive in $89 \%$ of HGSC, all LGSC, and in 27\% of EC, with no expression in clear cell carcinoma (CCC) and MC. Hormonal receptor expression, estrogen receptor (ER) was higher $(75 \%)$ in HGSC compared to other histotypes.

Table 1 Original diagnosis and IHC integrated revised ovarian cancer types

\begin{tabular}{|c|c|c|c|c|c|c|c|c|c|c|}
\hline & \multicolumn{7}{|c|}{ Revised diagnosis } & \multirow[b]{2}{*}{ Total } & \multirow[b]{2}{*}{ Concordance (\%) } & \multirow[b]{2}{*}{ Kappa } \\
\hline & $\mathrm{EPH}$ & GCT & LYMH & SCST & SC & Others & NM & & & \\
\hline \multicolumn{11}{|l|}{ Original Diagnosis } \\
\hline EPH & 123 & 9 & 0 & 9 & 0 & 0 & 6 & 147 & 83.7 & \\
\hline $\mathrm{GCT}$ & 3 & 17 & 0 & 1 & 0 & 0 & 0 & 21 & 81.0 & \\
\hline LMPH & 0 & 0 & 13 & 0 & 1 & 0 & 0 & 14 & 92.9 & 0.7019 \\
\hline SCST & 1 & 1 & 0 & 20 & 1 & 0 & 1 & 24 & 83.3 & \\
\hline SC & 0 & 0 & 0 & 0 & 2 & 0 & 0 & 2 & 100.0 & \\
\hline Others & 0 & 0 & 0 & 0 & 1 & 1 & 0 & 2 & 50.0 & \\
\hline Total & 127 & 27 & 13 & 30 & 5 & 1 & 7 & 210 & & \\
\hline Concordance (\%) & 96.6 & 63.0 & 100 & 66.7 & 40.0 & 100.0 & NA & & & \\
\hline
\end{tabular}

EPH Epithelia, GCT Germ Cell Tumors, LMPH Lymphomas, SCST Sex Cord Stromal Tumors, SC Sarcomas, NM Non Malignant 

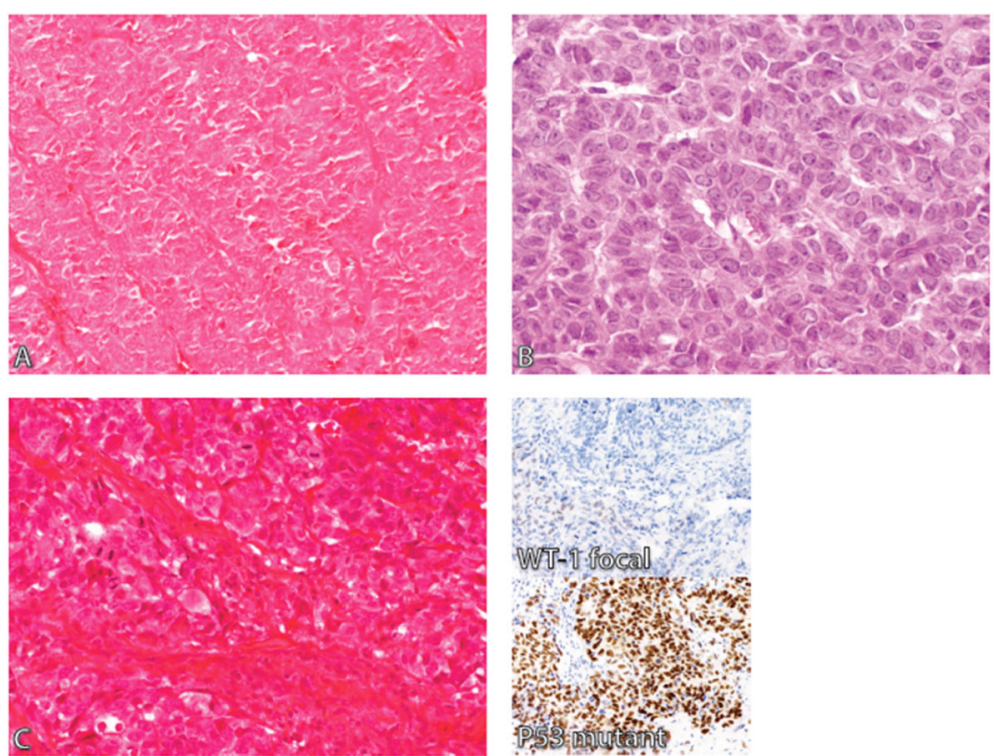

Fig. 2 The case (a) shows a poor morphology diagnosed as carcinoma NOS, and following re-stain (b), the morphology was that of adult granulosa cell tumor. $\mathbf{c}$ is another case that shows poor morphology with obvious mitotic figures diagnosed as carcinoma NOS, and IHC shows focal WT-1 stain and p53 mutant type staining pattern

For endometrioid carcinoma, ER and progesterone (PR) receptors were observed in 34 and $21 \%$ of cases, respectively, and one case of EC with mucinous features had a loss of MSH6 and ARID1A in tumor cells with a positive stain in stromal tissue and lymphocytes (See Fig. 3c). Abnormal staining of p53 was observed in $63 \%$ of HGSC, $18 \%$ of the EC, $20 \%$ of MC, and none of the LGSC. In sex cord stromal tumors, FOXL2 was expressed in 66 and 50\% of adult granulosa cell tumor (AGST) and juvenile granulosa cell tumor (JGCT), respectively. SATB2 expression was predictive of ovarian metastatic mucinous tumor $(p<$ 0.0001). Additionally, two cases of immature teratoma and one case of AGCT were positive for SATB2.
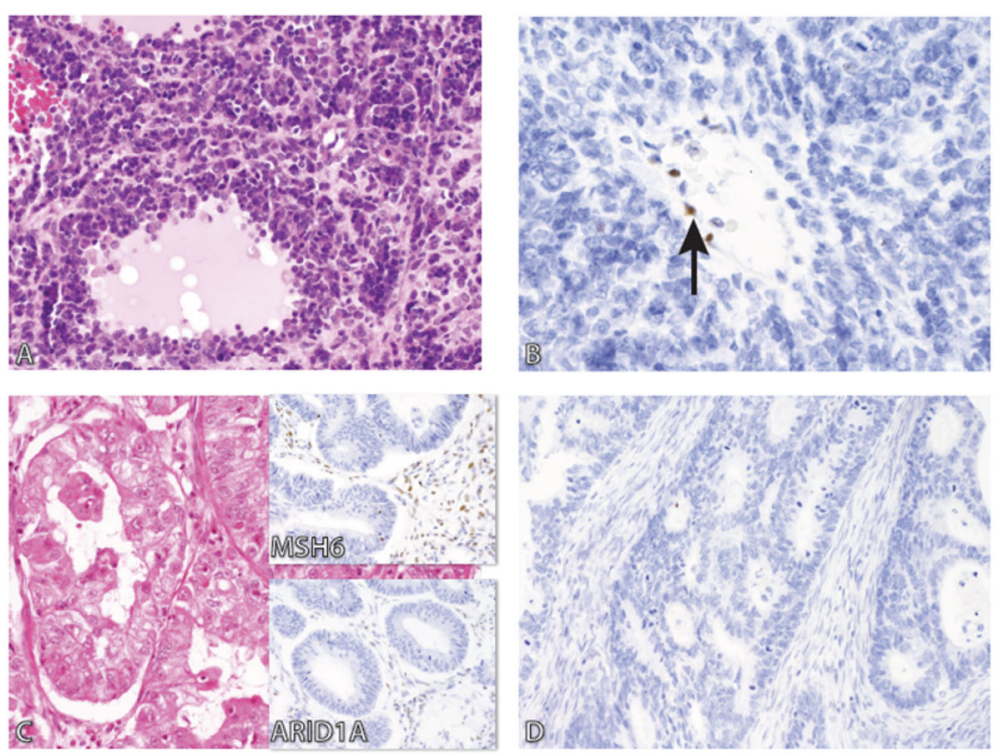

Fig. 3 A case of SCCOHT showing diffuse small cells with dark nuclei and pseudo follicles with eosinophilic material (a), and the loss of BRG1 staining in tumor cells (b) and positive staining of stroma cells (Arrow). c shows a case on EC with mucinous features (initially diagnosed as MC) with loss of MSH6 and ARID1A (insets) in tumor cells with positive staining in stromal tissue and lymphocytes. D is a case with Loss of ARID1A with no internal control (no staining of stromal tissue or lymphocytes) 
Table 2 Immunohistochemical marker expression across histotypes

\begin{tabular}{|c|c|c|c|c|c|c|c|}
\hline \multirow[t]{2}{*}{ Marker } & \multicolumn{5}{|c|}{ Carcinoma (\%) } & \multicolumn{2}{|c|}{ Sex Cord Stromal Tumors (\%) } \\
\hline & HGSC & EC & $\mathrm{CCC}$ & $M C$ & LGSC & $\mathrm{AGCT}$ & $\mathrm{JGCT}$ \\
\hline PAX8: Positive & 87.2 & 28.1 & 50 & 0 & 100 & 8.3 & 25 \\
\hline WT-1: Positive & 89.5 & 27.6 & 0 & 0 & 100 & 47.8 & 0 \\
\hline p53: Mutant & 63.2 & 18.8 & 0 & 20 & 0 & 4.2 & 0 \\
\hline p16: Abnormal & 75.4 & 53.1 & 50 & 60 & 50 & 54.2 & 75 \\
\hline Napsin-A: Positive & 0 & 3.1 & 25 & 0 & 0 & 0 & 0 \\
\hline ER: Positive & 75.4 & 34.4 & 25 & 10 & 50 & 37.5 & 50 \\
\hline PR: Positive & 19.3 & 21.9 & 0 & 0 & 0 & 33.3 & 0 \\
\hline ARID1A: Negative & 0 & 3.1 & 0 & 0 & 0 & 0 & 0 \\
\hline Vimentin: Diffuse & 14 & 21.9 & 25 & 0 & 50 & 95.8 & 75 \\
\hline MSH6: Negative & 0 & 3.1 & 0 & 0 & 0 & 0 & 0 \\
\hline PMS2: Negative & 0 & 0 & 0 & 0 & 0 & 0 & 0 \\
\hline FOXL2: Positive & 0 & 0 & 0 & 10 & 0 & 66.7 & 50 \\
\hline
\end{tabular}

For analysis of antibody performance, the known positive controls from APRL containing tonsil, Fallopian tube, placenta, and endometrium were included in each TMA. The performance of tested samples was assessed based on presence of internal controls for the tests, which require interpretation with internal controls (p53, ARID1A, MSH6, PMS2, and BRG1). All markers were not assessed equally across all the samples, as some samples were uninterpretable, or there was a loss of cores in the TMA. However, the majority of the samples were assessed for these markers, as shown in Table 3.

The most affected marker was ARID1A, whereby $47 \%$ of the cases had a loss of staining in tumor cells as well as stromal tissue and lymphocytes, which are the internal controls (See Fig. 3d), followed by PMS2 (12\%), and BRG1 (14\%). When we analyzed for the primary site of sample fixation in relation to loss of internal control, there was no significant difference when the samples were fixed in tertiary or non-tertiary hospitals. However, for ARID1A, most of the samples with positive controls had primary fixation in a tertiary hospital. These results are summarized in Table 3.

\section{Comparison of ovarian tumor types between east African and Alberta}

The comparison of ovarian cancer tumor types and histotypes distribution was performed using the following two populations: i) accessible ovarian cancer cases from East Africa diagnosed over a period of 15 years, and ii) Alberta Cancer Registry for cases diagnosed over a period of 9 years (2007 to 2016). In Alberta, the women population at risk of ovarian cancer is estimated to be 1.4 million women compared to the catchment area of the selected centers in East Africa of 9 million women at risk. There were 8-times more ovarian cancer cases in Alberta, compared to the selected population in East Africa, which is 6-times larger than the population of $\mathrm{Al}$ berta. This rough estimate suggests that there are 48 times more ovarian cancer cases in the Alberta population compared to the selected East African population. The distribution of the major tumor types in both populations showed epithelial tumors being the commonest, 89.9\% in Alberta, and 58\% in East Africa.

For the carcinomas, when a comparison was made by specific histotypes, HGSC occurred with the same frequency in both populations, as shown in Table 4. Still, $\mathrm{EC}$ was more common in the East Africa population

Table $3 \Vdash H C$ markers with no internal positive controls and level of the hospital

\begin{tabular}{|c|c|c|c|c|}
\hline \multirow[b]{2}{*}{ Marker } & \multirow[b]{2}{*}{ N (\%) } & \multicolumn{2}{|l|}{ Hospital level } & \multirow[b]{2}{*}{$\boldsymbol{p}$-value } \\
\hline & & Non-Tertiary $(\boldsymbol{N}=72)$ & Tertiary $(\boldsymbol{N}=147)$ & \\
\hline p53 $(N=204)$ & $18(8.8)$ & 3 & 15 & 0.178 \\
\hline ARID1A $(N=208)$ & 99 (47.6) & 50 & 49 & $0.000^{* *}$ \\
\hline MSH6 (N = 208) & $15(7.2)$ & 6 & 9 & 0.353 \\
\hline PMS2 $(N=206)$ & $26(12.6)$ & 9 & 17 & 0.913 \\
\hline BRG1 $(N=201)$ & $30(14.9)$ & 12 & 18 & 0.716 \\
\hline
\end{tabular}

**Majority of cases with positive internal control were from tertiary hospitals (87/109) 
Table 4 Patterns of ovarian cancer tumor types in Tanzania compared with Alberta, Canada

\begin{tabular}{|c|c|c|c|c|}
\hline & \multicolumn{4}{|l|}{ Major categories } \\
\hline & East Africa (n \%) & Mean age $( \pm \mathrm{SE})$ & Alberta (n \%) & Mean age $( \pm$ SE) \\
\hline Epithelial & $127(60.5)$ & $52.0(1.2)$ & 1645 (89.9) & $61.8(0.4)$ \\
\hline Germ cell tumor & 27 (12.6) & $24.2(2.7)$ & $58(3.2)$ & $31.2(1.5)$ \\
\hline Lymphoma & $13(6.2)$ & $15.3(3.7)$ & NA & NA \\
\hline Sex cord stromal tumor & $30(14.3)$ & $45.0(2.9)$ & $17(0.92)$ & $57.1(4.1)$ \\
\hline Sarcoma & $5(2.4)$ & $50.3(6.7)$ & $5(0.3)$ & $62.2(6.1)$ \\
\hline Others & $1(0.5)$ & $30.0(13.4)$ & $104(5.7)$ & $79.0(1.2)$ \\
\hline \multicolumn{5}{|l|}{ Histotypes specific } \\
\hline Carcinoma (epithelia) & $N=113$ & & $N=1646$ & \\
\hline HGSC & $57(50.4)$ & & $831(50.5)$ & \\
\hline $\mathrm{EC}$ & $32(28.3)$ & & $186(11.3)$ & \\
\hline $\mathrm{CCC}$ & $4(3.5)$ & & $132(8.0)$ & \\
\hline LGSC & $2(1.8)$ & & $15(0.9)$ & \\
\hline MC & $10(8.8)$ & & $120(7.3)$ & \\
\hline Others & $8(7.1)$ & & $362(22.0)$ & \\
\hline Germ cell tumors & $N=26$ & & $N=58$ & \\
\hline Dysgerminoma & $5(19.2)$ & & $17(29.3)$ & \\
\hline Yolk Sac Tumor & $14(53.8)$ & & $10(17.2)$ & \\
\hline Immature Teratoma & $4(15.4)$ & & $18(31.0)$ & \\
\hline Others & $3(11.6)$ & & $13(22.4)$ & \\
\hline Sex cord stromal tumor & $N=30$ & & $N=17$ & \\
\hline Adult granulosa Cell Tumor & $24(80.0)$ & & $12(70.6)$ & \\
\hline Sertoli Leydig Cell Tumor & $2(6.7)$ & & $4(23.5)$ & \\
\hline Juvenile Granulosa Cell Tumor & $4(13.3)$ & & 0 & \\
\hline Others & 0 & & $1(5.9)$ & \\
\hline
\end{tabular}

than the Alberta population $(28.3 \%$ versus $11.3 \%$ respectively).

There was no striking difference for CCC, LGSC, and $\mathrm{MC}$, and these cases occurred at a lower frequency in both populations. For Germ Cell Tumors, there was a predominance of Dysgerminoma and Immature Teratoma in the Alberta population (29.3 and 31\%, respectively), compared to the East Africa population (19.2 and $15.4 \%$ respectively). However, the Yolk Sac Tumors were more common in East Africa compared to Alberta (53.8 and $17.2 \%$, respectively). The Adult Granulosa Cell Tumor was the commonest sex cord stromal tumor in both East Africa and Alberta populations (80 and 70.6\%, respectively), though in relatively low numbers in $\mathrm{Al}$ berta compared to the numbers of ovarian cancer cases. Juvenile Granulosa Cell tumors were exclusively seen in East Africa in $13.3 \%$ of the cases of SCST.

\section{Discussion}

This study shows extremely few cases of ovarian cancer in East Africa compared to the Alberta population. There were limitations, and likely but unknown bias influencing the results. The agestandardized incidence rate could not be calculated for the East African population because of the lack of census data. On the one hand, a large proportion of the female population in East Africa may not yet be at the age of risk for ovarian cancer. On the other hand, the proportion of older females who carry the highest risk is likely to be lower in East Africa compared to Alberta [12]. The WHO 2014 cancer country profiles show that the life expectancy of Canadian women is 20 years higher compared to East African women. The larger population per cancer center in East Africa and other impediments such as access to health care facilities will most likely result in underdiagnosis of cases. For example, older women in rural areas may die of undiagnosed disease, unaware of the significance of the symptoms, and lacking the resources to seek medical attention [9]. While our data provide the first benchmark of ovarian cancer in East Africa, these limitations lead us to assume that cases 
from selected areas in East Africa may not be representative of the whole population. Therefore, there is a need to establish cancer registries and census data in Eastern Africa, similar to those in developed countries.

Ovarian cancer shows a wide geographical variation with a higher incidence in North America compared to Asia and Africa [13]. In this study, the Alberta population had more cases of epithelial cancer (89\%) compared to East Africa (60\%). In contrast, germ cell tumors and sex cord stromal tumors were more common in East Africa compared to the Alberta population. A large population-based study (CONCORD-2) reported a high proportion of germ cell tumors in Asia (4.2\%), compared to Europe (1.3\%) and North America $(2.0 \%)$. In this study, we report a high proportion of germ cell tumors in East Africa (12.5\%), similar to the one reported in Russia (11.4\%) [14]. Similarly, the proportion of sex cord stromal tumors was $14.3 \%$ for East Africa, and $0.92 \%$ for the Alberta population. Because germ cell and sex cord stromal tumors occur at a younger age, this could reflect the age distribution of the underlying population and selection bias. There is a possibility that younger women with germ cell tumor or sex cord stromal tumor might seek medical attention and receive treatment, while older women with advanced epithelial cancer may not.

Morphology-based diagnosis of cancer remains a mainstay in developing countries, and ancillary immunohistochemical tests are rarely available. The diagnostic accuracy of ovarian cancer in East Africa showed a substantial agreement with the diagnosis of major categories of ovarian cancer but was only fair for specific histotypes. This agreement is a remarkable fact, given the inadequate laboratory resources in these settings, compared to North America, exemplified by the tremendous differences in the quality of the basic $H \& E$ stains. The fact that pathologists in East Africa can make a highly accurate diagnosis with virtually zero contrast $H \& E$ stains is an example of the remarkable adaption and ingenuity of the human brain. There is no reason to believe that they could not achieve a similar diagnostic accuracy regarding more specific histotyping with simple standardization of the H\&E staining protocol.

In the past 15 years, there has been a significant change in the understanding and diagnosis of ovarian cancer, such that histotype has emerged as an important prognostic and predictive marker $[15,16]$. Historically, the reproducibility of diagnosis of ovarian carcinoma based on the cell types [17-19] was low, but nowadays, the inter-observer reproducibility is excellent, and if not, it can be improved by the use of ancillary immunohistochemistry tests [20]. In this study, the morphological diagnosis of ovarian carcinoma was only fair, likely a result of a failure to use the current criteria for tumor cell types in routine practice [21], and the majority of the cases of carcinoma were unspecified. Significant numbers of unspecified carcinomas $(\sim 43 \%)$ in this study were reclassified as HGSC in a revised diagnosis, and these are the diagnoses likely made before 2014, where serous carcinomas were separated into HGSC and LGSC. For instance, a study performed in Canada necessitated a review of diagnoses; the majority (78\%) of unspecified carcinomas based on 2003 WHO classification were reclassified to HGSC [22]. The problematic areas in ovarian carcinoma histotyping remain the differentiation of HGSC versus high grade EC and vice versa [6, $20,23]$. In the current study, $29 \%$ of the EC were reclassified to HGSC, contrary to $17 \%$ in a study carried out in Canada [22].

Mucinous carcinoma showed a poor concordance between the original diagnosis and the revised diagnosis, and a significant number (26\%) were reclassified to EC, whereas $17 \%$ were metastatic gastrointestinal neoplasms. Differentiating primary ovarian MC from metastatic adenocarcinomas has been an area of challenge. In previous years, the diagnosis of $\mathrm{MC}$ was made frequently, constituting up to $14 \%$ of ovarian carcinoma [24]. Currently, with an improved understanding of ovarian carcinoma histotypes, and the use of ancillary tests, MC has become a rare subset of ovarian carcinoma, making it less than $5 \%$ of the cases [25-27]. In this study, we used SATB2, a recently identified marker, for differentiating primary ovarian from secondary colorectal/appendiceal tumors $[28,29]$. The tumors which showed mucinous, or endometrioid like morphology suspicious for metastasis with an expression of SATB2 were considered as metastatic.

In this study, the cases of $\mathrm{MC}$, which were reclassified as $\mathrm{EC}$, were probably related to a failure of recognition of confirmatory endometrioid features or overreliance on features like mucinous differentiation, which do occur in EC [30, 31]. Furthermore, mucinous carcinoma with unapparent intracytoplasmic mucin can also mimic EC [27], and two cases which were initially diagnosed as CCC were actually endometrioid carcinoma with clear cell changes [30].

For non-epithelia tumors, the reproducibility in assigning tumor types was substantial, with the highest concordance in lymphomas (92\%), followed by sex cord stromal tumors (83\%), and germ cell tumors $81 \%$. We did not go into specific histological types in these smaller categories because of lower case numbers but noted a high number of primary ovarian lymphomas.

This study identified one case of ovarian small cell carcinoma hypercalcemic type (SCCOHT), which was 
originally diagnosed as neuroblastoma. This lethal cancer, which affects young women, is characterized by an aggressive clinical course, poor prognosis, and a recently discovered pathognomonic mutation in SMARCA4 [32-34]. The reasons for such diagnosis could be the morphology of a round dark cell tumor, which can easily attract a differential diagnosis of neuroblastoma in a setting where IHC tests are not routinely performed. Yet, ovarian neuroblastoma at the age of 30 years is quite uncommon, although it has been reported as primary ovarian or associated with mature teratoma in a few cases $[35,36]$. Lack of awareness for the existence of this diagnosis in the current classification was a likely contributing factor, as well as the rarity of this tumor, noting that most of the pathologists would see at most one such case during their practice [21]. Foremost, this case illustrates the inability to render a correct diagnosis without access to confirmatory molecular testing for molecularly defined rare cancers.

This study applied various IHC markers used in ovarian cancer diagnosis and histotyping. The expression patterns followed the expected trend with some differences. At current, TP53 mutations are ubiquitously present in HGSC $[37,38]$, and optimized p53 immunohistochemistry is used as a surrogate marker of TP53 mutation $[38,39]$. A notable difference was seen in the frequency of mutant p53 staining; only $63 \%$ of HGSC had mutant p53, whereas, in EC, it was seen in $18 \%$ of the cases as expected [22]. The low rate of abnormal p53 seen in HGSC could be due to various reasons. Some cases lost internal control, which was even commonly observed with ARID1A. In this study, we used a p53 optimized antibody, and a standardized staining platform, as well as known positive and negative controls. Therefore, poor staining and loss of internal control cannot be explained by the analytical process; the issue probably occurred in pre-analytical phases specifically related to tissue fixation. In East Africa, there is no uniform, standardized protocol for tissue fixation. The samples are sent from various hospitals to the pathology centres, and the quality of formalin and time for fixation vary considerably. Furthermore, FFPE tissue blocks are not stored in a controlled environment.

Hormonal receptor expression in EC was also lower compared to the previous studies [40, 41], and this could probably be attributed to weak staining or biological behavior of the EC, which cannot be justified. Low hormonal receptor expression is common in high grade EC [42], but, in this study, tumor grades were equally distributed across all EC cases. Interestingly, we observed only one case $(3 \%)$ (41-year-old patient with endometrioid carcinoma) that showed abnormal mismatch repair, contrary to $13 \%$, as reported previously [43]. MSH6 was lost, indicating probably Lynch syndrome. This loss suggests that Lynch Syndrome likely exists in East Africa, but more extensive studies on endometrioid carcinomas are needed to assess its prevalence in the East African population.

\section{Conclusion}

Our data suggest an extremely low number of ovaria cancer in selected East African populations compared to the Alberta population. The main limitation was a likely but unknown selection bias. Correct ovarian carcinoma histotyping is vital as some low stage patients could be spared by aggressive chemotherapy. In a resourcelimited setting, simple improvement of the basic H\&E stain could have a substantial impact on accurate diagnosis according to current WHO criteria. Diagnosis of rare molecularly defined entities requiring access to molecular testing remains a challenge for pathologists in a resource-limited setting and calls for solidarity from resource-rich countries.

\section{Supplementary information}

Supplementary information accompanies this paper at https://doi.org/10. 1186/s13000-020-01000-3.

Additional file 1: Supplementary table 1. Immunohistochemical protocols.

Additional file 2: Supplementary table 2. Concordance of the correctly classified original diagnosis (Kappa $=0.3430,96 \%$ Cl: $0.2774-$ 0.4087).

\section{Abbreviations}

AGCT: Adult granulosa cell tumor; BMC: Bugando Medical Center; CCC: Clear cell carcinoma; EC: Endometrioid carcinoma; EPH: Epithelia; FFPE: Formalin fixed paraffin embedded; GCT: Germ cell tumors; HGSC: High grade serous carcinoma; IHC: Immunohistochemistry; JGSC: Juvenile granulosa cell tumor; KCMC: Kilimanjaro Christian Medical Center; LGSC: Low grade serous carcinoma; LMPH: Lymphomas; MC: Mucinous carcinoma; NM: Non malignant; SCST: Sex-cord stromal tumors; SCCOHT: Small cell carcinoma of the ovary, hypercalcemic type; TMA: Tissue microarray; WHO: World Health Organization

\section{Acknowledgements}

Technical assistance provided by Dr. Shuhong Liu is greatly appreciated, as well as Ms. Esther Reuben and Ms. Aquilina Pangani from Bugando Medical Center, Pathology Department for the acquisition of study materials, and Professor Rebert Peck for english editing.

\section{Authors' contributions}

PR, MK -Design, data acquisition, analysis and manuscript preparation. DTData acquisition, analysis, and manuscript preparation. AM, RL, WM-Data ac quisition, and manuscript preparation. The authors read and approved the final manuscript.

\section{Funding}

No funding was received.

Availability of data and materials

Submitted as additional files.

\section{Ethics approval}

The study received ethical clearance CREC/193/2017 from Joint CUHAS/BMC Ethics \& Review Committee and HREBA.CC-16-0913 from Alberta, Canada. 


\section{Consent for publication}

Not applicable. All authors read and approved the final manuscript.

\section{Competing interests}

All authors declared no conflict of interest.

\section{Author details}

'Department of Pathology, Catholic University of Health and Allied Sciences (CUHAS), Box 1464, Mwanza, Tanzania. ${ }^{2}$ Department of Pathology and Laboratory Medicine, Foothill Medical Center, University of Calgary, 1403 - 29 Street NW, Calgary, AB, Canada. ${ }^{3}$ Holy Cross Cancer Center, 2210 2nd St. SW, Calgary, AB, Canada. ${ }^{4}$ Department of Pathology, Kilimanjaro Christian Medical Center, Box 3010, Moshi, Tanzania. ${ }^{5}$ Department of Pathology, College of Health Sciences Makerere University, Box. 7072, Kampala, Uganda. ${ }^{6}$ Department of Pathology, Mbeya Referral Hospital, Box 419, Mbeya, Tanzania.

Received: 6 May 2020 Accepted: 8 July 2020

\section{Published online: 16 July 2020}

\section{References}

1. Coleman MP, Esteve J, Damiecki P, Arslan A, Renard H. Trends in cancer incidence and mortality. IARC Sci Publ. 1993;121:1-806.

2. Stefan DC, Elzawawy AM, Khaled HM, Ntaganda F, Asiimwe A, Addai BW, et al. Developing cancer control plans in Africa: examples from five countries. Lancet Oncol. 2013;14(4):e189-95.

3. Stefan DC. Cancer Care in Africa: an overview of resources. J Glob Oncol. 2015;1(1):30-6.

4. Korir A, Okerosi N, Ronoh V, Mutuma G, Parkin M. Incidence of cancer in Nairobi, Kenya (2004-2008). Int J Cancer. 2015;137(9):2053-9.

5. Sung PL, Chang YH, Chao KC, Chuang CM. Task force on systematic R, meta-analysis of ovarian C. global distribution pattern of histological subtypes of epithelial ovarian cancer: a database analysis and systematic review. Gynecol Oncol. 2014;133(2):147-54.

6. Gilks CB, lonescu DN, Kalloger SE, Kobel M, Irving J, Clarke B, et al. Tumor cell type can be reproducibly diagnosed and is of independent prognostic significance in patients with maximally debulked ovarian carcinoma. Hum Pathol. 2008:39(8):1239-51.

7. Kanavos $P$. The rising burden of cancer in the developing world. Ann Oncol. 2006;17(Suppl 8):viii15-23.

8. African Pathologists' Summit Working G. Proceedings of the African pathologists summit; march 22-23, 2013; Dakar, Senegal: a summary. Arch Pathol Lab Med. 2015;139(1):126-32.

9. Rambau PF. Pathology practice in a resource-poor setting: Mwanza. Tanzania Arch Pathol Lab Med. 2011;135(2):191-3.

10. Adesina A, Chumba D, Nelson AM, Orem J, Roberts DJ, Wabinga $H$, et al. Improvement of pathology in sub-Saharan Africa. Lancet Oncol. 2013;14(4) e152-7.

11. Kobel M, Rahimi K, Rambau PF, Naugler C, Le Page C, Meunier L, et al. An Immunohistochemical algorithm for ovarian carcinoma typing. Int J Gynecol Pathol. 2016;35(5):430-41.

12. Jayson GC, Kohn EC, Kitchener HC, Ledermann JA. Ovarian cancer. Lancet. 2014:384(9951):1376-88

13. Ferlay J, Shin HR, Bray F, Forman D, Mathers C, Parkin DM. Estimates of worldwide burden of cancer in 2008: GLOBOCAN 2008. Int J Cancer. 2010; 127(12):2893-917

14. Matz M, Coleman MP, Sant M, Chirlaque MD, Visser O, Gore M, et al. The histology of ovarian cancer: worldwide distribution and implications for international survival comparisons (CONCORD-2). Gynecol Oncol. 2017; 144(2):405-13.

15. Gilks CB, Prat J. Ovarian carcinoma pathology and genetics: recent advances. Hum Pathol. 2009:40(9):1213-23.

16. Gilks CB, Kommoss F. Ovarian carcinoma Histotypes: their emergence as important prognostic and predictive markers. Oncology (Williston Park). 2016;30(2):178-9.

17. Cramer SF, Roth LM, Ulbright TM, Mazur MT, Nunez CA, Gersell DJ, et al. Evaluation of the reproducibility of the World Health Organization classification of common ovarian cancers. With emphasis on methodology. Arch Pathol Lab Med. 1987;111(9):819-29.
18. Hernandez E, Bhagavan BS, Parmley TH, Rosenshein NB. Interobserver variability in the interpretation of epithelial ovarian cancer. Gynecol Oncol. 1984;17(1):117-23.

19. Lund B, Thomsen HK, Olsen J. Reproducibility of histopathological evaluation in epithelial ovarian carcinoma. Clinical implications. APMIS. 1991 99(4):353-8.

20. Kobel M, Kalloger SE, Baker PM, Ewanowich CA, Arseneau J, Zherebitskiy V, et al. Diagnosis of ovarian carcinoma cell type is highly reproducible: a transcanadian study. Am J Surg Pathol. 2010;34(7):984-93.

21. Singh N, Gilks CB. The changing landscape of gynaecological cancer diagnosis: implications for histopathological practice in the 21st century. Histopathology. 2017;70(1):56-69.

22. Kobel M, Luo L, Grevers X, Lee S, Brooks-Wilson A, Gilks CB, et al. Ovarian carcinoma Histotype: strengths and limitations of integrating morphology with Immunohistochemical predictions. Int J Gynecol Pathol. 2019;38(4): 353-62.

23. Al-Hussaini M, Stockman A, Foster H, McCluggage WG. WT-1 assists in distinguishing ovarian from uterine serous carcinoma and in distinguishing between serous and endometrioid ovarian carcinoma. Histopathology. 2004;44(2):109-15

24. Schiavone MB, Herzog TJ, Lewin SN, Deutsch I, Sun X, Burke WM, et al. Natural history and outcome of mucinous carcinoma of the ovary. Am J Obstet Gynecol. 2011;205(5):480 e1-8.

25. Kobel M, Kalloger SE, Huntsman DG, Santos JL, Swenerton KD, Seidman JD, et al. Differences in tumor type in low-stage versus high-stage ovarian carcinomas. Int J Gynecol Pathol. 2010;29(3):203-11.

26. Seidman JD, Horkayne-Szakaly I, Haiba M, Boice CR, Kurman RJ, Ronnett BM The histologic type and stage distribution of ovarian carcinomas of surface epithelial origin. Int J Gynecol Pathol. 2004;23(1):41-4.

27. Koonings PP, Campbell K, Mishell DR Jr, Grimes DA. Relative frequency of primary ovarian neoplasms: a 10-year review. Obstet Gynecol. 1989;74(6): 921-6.

28. Moh M, Krings G, Ates D, Aysal A, Kim GE, Rabban JT. SATB2 expression distinguishes ovarian metastases of colorectal and Appendiceal origin from primary ovarian tumors of mucinous or Endometrioid type. Am J Surg Pathol. 2016:40(3):419-32.

29. Meagher NS, Wang L, Rambau PF, Intermaggio MP, Huntsman DG, Wilkens LR, et al. A combination of the immunohistochemical markers CK7 and SATB2 is highly sensitive and specific for distinguishing primary ovarian mucinous tumors from colorectal and appendiceal metastases. Mod Pathol. 2019:32(12): 1834-46.

30. Soslow RA. Histologic subtypes of ovarian carcinoma: an overview. Int J Gynecol Pathol. 2008;27(2):161-74.

31. Ramalingam P. Morphologic, Immunophenotypic, and molecular features of epithelial ovarian Cancer. Oncology (Williston Park). 2016;30(2):166-76.

32. Young RH, Oliva E, Scully RE. Small cell carcinoma of the ovary, hypercalcemic type. A clinicopathological analysis of 150 cases. Am J Surg Pathol. 1994;18(11):1102-16.

33. Kupryjanczyk J, Dansonka-Mieszkowska A, Moes-Sosnowska J, PlisieckaHalasa J, Szafron L, Podgorska A, et al. Ovarian small cell carcinoma of hypercalcemic type - evidence of germline origin and SMARCA4 gene inactivation. A pilot study. Pol J Pathol. 2013;64(4):238-46.

34. Witkowski L, Carrot-Zhang J, Albrecht S, Fahiminiya S, Hamel N, Tomiak E, et al. Germline and somatic SMARCA4 mutations characterize small cell carcinoma of the ovary, hypercalcemic type. Nat Genet. 2014;46(5):438-43.

35. Niwa Y, Yamamuro O, Kato N, Tsuzuki T. Two cases of primary ovarian neuroblastoma arising from mature cystic teratomas. Gynecol Oncol Case Rep. 2013;5:58-60

36. Singh M, Arora P, Singh S, Bohara S, Khurana N, Zutshi V, et al. Adult neuroblastoma of the ovary: a rare tumor with review of literature. J Cancer Res Ther. 2010;6(3):367-9.

37. Ahmed AA, Etemadmoghadam D, Temple J, Lynch AG, Riad M, Sharma R, et al. Driver mutations in TP53 are ubiquitous in high grade serous carcinoma of the ovary. J Pathol. 2010;221(1):49-56.

38. Na K, Sung JY, Kim HS. TP53 mutation status of Tubo-ovarian and peritoneal high-grade serous carcinoma with a wild-type p53 Immunostaining pattern. Anticancer Res. 2017;37(12):6697-703

39. Kobel M, Piskorz AM, Lee S, Lui S, LePage C, Marass F, et al. Optimized p53 immunohistochemistry is an accurate predictor of TP53 mutation in ovarian carcinoma. J Pathol Clin Res. 2016:2(4):247-58. 
40. Sieh W, Kobel M, Longacre TA, Bowtell DD, deFazio A, Goodman MT, et al. Hormone-receptor expression and ovarian cancer survival: an ovarian tumor tissue analysis consortium study. Lancet Oncol. 2013;14(9):853-62.

41. Rambau P, Kelemen LE, Steed H, Quan ML, Ghatage P, Kobel M. Association of Hormone Receptor Expression with Survival in Ovarian Endometrioid Carcinoma: Biological Validation and Clinical Implications. Int J Mol Sci. 2017;18(3):515

42. Assem H, Rambau PF, Lee S, Ogilvie T, Sienko A, Kelemen LE, et al. Highgrade Endometrioid carcinoma of the ovary: a Clinicopathologic study of 30 cases. Am J Surg Pathol. 2018;42(4):534-44.

43. Rambau PF, Duggan MA, Ghatage P, Warfa K, Steed H, Perrier R, et al. Significant frequency of MSH2/MSH6 abnormality in ovarian endometrioid carcinoma supports histotype-specific Lynch syndrome screening in ovarian carcinomas. Histopathology. 2016;69(2):288-97.

\section{Publisher's Note}

Springer Nature remains neutral with regard to jurisdictional claims in published maps and institutional affiliations.

Ready to submit your research? Choose BMC and benefit from:

- fast, convenient online submission

- thorough peer review by experienced researchers in your field

- rapid publication on acceptance

- support for research data, including large and complex data types

- gold Open Access which fosters wider collaboration and increased citations

- maximum visibility for your research: over $100 \mathrm{M}$ website views per year

At $\mathrm{BMC}$, research is always in progress.

Learn more biomedcentral.com/submissions 